\title{
EDITORIAL
}

\section{EL COSTE DEL CÁNCER LABORAL EN ESPAÑA}

\section{Manolis Kogevinas}

Centre de Recerca en Epidemiologia Ambiental (CREAL)

Los accidentes de trabajo y las enfermedades asociadas con el trabajo constituyen un porcentaje importante de la morbilidad y mortalidad en la población. Durante las últimas décadas en Europa y España se han aplicado medidas de control de las exposiciones laborales y esto ha tenido como repercusión el descenso de la mortalidad asociada a exposiciones laborales. Sin embargo, las enfermedades producidas por exposiciones en el lugar de trabajo aún producen un coste alto para los trabajadores, los empleadores y la sociedad.

Montse García-Gómez y colaboradores ${ }^{1}$ publican en esta revista una estimación del porcentaje de cánceres de pulmón y vejiga atribuidos a exposiciones laborales y hacen una estimación de los costes para los servicios de salud del diagnóstico y tratamiento de los dos tumores más frecuentes asociados a ellas, los de pulmón y vejiga urinaria. Su trabajo, aunque no contempla todos los costes para la sociedad de estos dos tumores, pone la base para un análisis muy poco frecuente en España y que es absolutamente necesario.

Los cancerígenos laborales ocupan un puesto especial en la identificación y prevención del cáncer ${ }^{2}$. Han sido de los primeros cancerígenos humanos identificados y aún actualmente una proporción importante de los identificados son de origen laboral. En Inglaterra, en

Correspondencia

Centre de Recerca en Epidemiologia Ambiental (CREAL)

IMIM (Hospital del Mar Research Institute)

Dr Aiguader, 88

Barcelona 08003

kogevinas@creal.cat

www.creal.cat
1771, Percival Pott, en su obra Chirurgical works, describió una mayor frecuencia de cáncer en el escroto de los deshollinadores y se asoció con la exposición al hollín. El cáncer de vejiga, que es uno de los principales tumores atribuibles a exposiciones laborales, ocupa un lugar importante en la historia de la epidemiología del cáncer de orígen profesional, ya que la mayor incidencia acumulada de cualquier tipo de cáncer es la del de vejiga entre los trabajadores de la industria de colorantes. Además, todos ellos deben ser considerados como prevenibles y para ello es esencial su identificación. En base a las evaluaciones de la Agencia Internacional de Investigación sobre el Cáncer (IARC), hay 400 agentes químicos o físicos que se pueden considerar como cancerígenos y 150 se dan en el lugar de trabajo. Además, 18 ocupaciones o industrias están asociadas a un aumento del riesgo de cáncer entre sus trabajadores. En países industrializados aproximadamente un cuarto de todas las defunciones son por cáncer. Los tumores que han sido asociados más frecuentemente a exposiciones laborales son los de pulmón, vejiga urinaria, cavidad nasal, hígado (angiosarcoma), mesotelioma, leucemia, linfomas y cáncer de piel no melanocítico. Se ha descrito también una asociación entre exposiciones industriales y otros cánceres, como por ejemplo el de páncreas, cerebro, laringe, próstata, colon, riñón, así como sarcomas de tejidos blandos, mielomas y otros, pero las evidencias no son tan claras.

Hay una cierta controversia en relación al porcentaje de todos los cánceres que se pueden atribuir a exposiciones laborales. La gran 
mayoría de los investigadores y agencias evaluadoras consideran que alrededor del 5\% de todos los cánceres se puede atribuir directamente a exposiciones que se consideran laborales $^{3}$. Otros investigadores consideran que dicha proporción es más elevada. GarcíaGómez publica una estimación del porcentaje de cánceres de pulmón y vejiga atribuidos a exposiciones laborales e indica que casi 4.000 hospitalizaciones por cáncer de pulmón y 2.400 por cáncer de vejiga urinaria se pueden atribuir a exposiciones laborales. Aunque la prevención del cáncer laboral ha sido uno de los grandes éxitos de la salud pública y laboral en los países industrializados, en los últimos años continúa habiendo un porcentaje importante de cánceres. Unas de las estimaciones más completas que existen son las detalladas para el cáncer en Reino Unido ${ }^{4}$, que indican que en la actualidad las exposiciones laborales continúan provocando el $4 \%$ de todos los cánceres, el mismo porcentaje estimado hace 30 años por Doll y Peto 5 .

García-Gómez y colaboradores ${ }^{1}$ indican el grave problema en España con el reconocimiento de los cánceres de origen laboral, dado que las enfermedades profesionales apenas aparecen en los registros oficiales. Se ha estimado que el $83 \%$ de las enfermedades profesionales no son reconocidas como tales. A pesar de que en el listado de enfermedades profesionales español se incluyen numerosos agentes que están relacionados con determinados cánceres, el número de estos notificados como de origen laboral es prácticamente inexistente.

García-Gómez propone también una estimación de los costes directos de los cánceres de pulmón y de vejiga de origen laboral y calculan que dichos costes pueden ser estimados en 88 millones de Euros por año para su diagnóstico y tratamiento. Existen aspectos éticos, legales y sociales cuando se evalúan los efectos y la prevención de dichas exposiciones. Además se debe utilizar el parámetro de coste beneficio $^{6}$. Las consecuencias económicas de la morbilidad laboral solo se han investigado en años recientes. Múltiples estudios indican sin ninguna duda el enorme coste directo de dichas enfermedades en los servicios de salud, pero también los costes indirectos por el absentismo, las perdidas en la producción laboral y la discapacidad ${ }^{7}$. En estos últimos años se están desarrollando estudios de coste efectividad y coste beneficio evaluando las intervenciones en el lugar del trabajo. Las estimaciones presentadas por García-Gómez son las primeras que se hacen en España y subrayan la importancia del problema del cáncer laboral para los servicios sanitarios.

\section{BIBLIOGRAFÍA}

1. García Gómez M, Urbanos Garrido R, Castañeda López R, López Menduiña P. Costes sanitarios directos de las neoplasias de pulmón y vejiga de origen laboral en España en 2008. Rev Esp Salud Pública. 2012; 86: 127-38.

2. Kogevinas M, Harrington M, Vermeulen R. Occupational cancer: Epidemiology, biological mechanisms and biomarkers. Chapter 35. En: Hunter's Diseases of Occupations. Editors: PJ Baxter, PH Adams, T-C Aw, A Cockcroft, JM Harrington. London: Hodder Arnold Publication; 2011

3. Kogevinas M et al. Cáncer laboral en España. Valencia: Instituto Sindical de Trabajo, Ambiente y Salud (ISTAS); 2005.

4. Rushton L, Hutchings S, Brown T. The burden of cancer at work: estimation as the first step to prevention. Occup Environ Med. 2008; 65:789-800.

5. Doll R, Peto R. The causes of cancer: quantitative estimates of avoidable risks of cancer in the United States today. J Natl Cancer Inst. 1981; 66: 1191-1308.

6. Murray CJ, Evans DB, Acharya A, et al. Development of WHO guidelines on generalized cost-effectiveness analysis. Health Econ. 2000;9(3):235-251.

7. Burdorf A. Economic evaluation in occupational health: its goals, challenges, and opportunities. Scand J Work Environ Health 2007;33:161-4. 